ARTICLE

https://doi.org/10.1038/s41467-020-14415-9

\title{
Tissue $\mathrm{pO}_{2}$ distributions in xenograft tumors dynamically imaged by Cherenkov-excited phosphorescence during fractionated radiation therapy
}

\author{
Xu Cao (1) 1,2, Srinivasa Rao Allu (10 3,4, Shudong Jiang 1,5, Mengyu Jia1, Jason R. Gunn', Cuiping Yao ${ }^{1,6}$, \\ Ethan P. LaRochelle (1) ${ }^{1}$, Jennifer R. Shell', Petr Bruza1, David J. Gladstone1,5,7, Lesley A. Jarvis ${ }^{5,7}$, Jie Tian 2,8, \\ Sergei A. Vinogradov ${ }^{3,4 \star} \&$ Brian W. Pogue ${ }^{1,5 \star}$
}

Hypoxia in solid tumors is thought to be an important factor in resistance to therapy, but the extreme microscopic heterogeneity of the partial pressures of oxygen $\left(\mathrm{pO}_{2}\right)$ between the capillaries makes it difficult to characterize the scope of this phenomenon without invasive sampling of oxygen distributions throughout the tissue. Here we develop a noninvasive method to track spatial oxygen distributions in tumors during fractionated radiotherapy, using oxygen-dependent quenching of phosphorescence, oxygen probe Oxyphor PtG4 and the radiotherapy-induced Cherenkov light to excite and image the phosphorescence lifetimes within the tissue. Mice bearing MDA-MB-231 breast cancer and FaDu head neck cancer xenografts show different $\mathrm{pO}_{2}$ responses during each of the 5 fractions ( 5 Gy per fraction), delivered from a clinical linear accelerator. This study demonstrates subsurface in vivo mapping of tumor $\mathrm{pO}_{2}$ distributions with submillimeter spatial resolution, thus providing a methodology to track response of tumors to fractionated radiotherapy.

\footnotetext{
${ }^{1}$ Thayer School of Engineering, Dartmouth College, Hanover, NH, USA. ${ }^{2}$ Engineering Research Center of Molecular and Neuro Imaging of Ministry of Education, School of Life Science and Technology, Xidian University, Xi'an, Shaanxi, China. ${ }^{3}$ Department of Biochemistry and Biophysics, Perelman School of Medicine, University of Pennsylvania, Philadelphia, PA, USA. ${ }^{4}$ Department of Chemistry, School of Arts and Sciences, University of Pennsylvania, Philadelphia, PA, USA. ${ }^{5}$ Norris Cotton Cancer Center, Dartmouth-Hitchcock Medical Center, Lebanon, NH, USA. ${ }^{6}$ Key Laboratory of Biomedical Information Engineering of Ministry of Education, Institute of Biomedical Analytical Technology and Instrumentation, School of Life Science and Technology, Xi'an Jiaotong University, Xi'an, Shaanxi, China. ${ }^{7}$ Department of Medicine, Geisel School of Medicine, Dartmouth College, Hanover, NH, USA. ${ }^{8}$ CAS Key Laboratory of Molecular Imaging, Beijing Key Laboratory of Molecular Imaging, The State Key Laboratory of Management and Control for Complex Systems, Institute of Automation, Chinese Academy of Sciences, Beijing, China. *email: vinograd.upenn@gmail.com; brian.w.pogue@dartmouth.edu
} 
ow levels of partial pressure of oxygen $\left(\mathrm{pO}_{2}\right)$ in tissue, commonly referred to as hypoxia, are known to reduce the potential for therapeutic radiation damage, inducing tumor resistance and facilitating tumor cells escape from treatment ${ }^{1-3}$. Meta-analyses reveal that improvements in regional control of cancer are primarily observed in patients with well-oxygenated tumors and not in patients with hypoxic tumors ${ }^{4,5,6}$. Attempts have been made in the past in clinical trials to reduce hypoxia by administration of carbogen and nicotinamide 7 or to provide radiation boost in the hypoxic areas ${ }^{8}$, although no methodology has yet been widely adopted in clinics. A part of the problem is that the progress in understanding and clinically addressing consequences of hypoxia has been limited by the lack of methods for non-invasive, fast, and repeatable mapping of $\mathrm{pO}_{2}$ distributions in tumor tissues. Multiple-track oxygen electrode measurements ${ }^{9}$ or ex vivo tissue immunohistochemistry ${ }^{10}$ suffer from invasiveness, which limits their clinical utility. Macroscopic assessment of tumor oxygenation in humans has been performed by positron emission tomography (PET) with fluoromisonidazole (FMISO) hypoxia tracer ${ }^{11,12}$ and by blood oxygen level dependent magnetic resonance imaging (BOLD MRI) ${ }^{13,14}$. However, these macroscopic imaging modalities are not able to fully sample the heterogeneity of the tumor microenvironment, while both chronic and transient hypoxia are documented to be microregional $^{15}$. Additionally, measurements by BOLD MRI report on changes in the vascular $\mathrm{pO}_{2}$, whereas in tissues with abnormally developed vasculature, such as tumors, blood oxygen levels may be only poorly correlated with extravascular "tissue" $\mathrm{pO}_{2}$, and tissue oxygen may be misrepresented. Hence, measurements of intratumoral $\mathrm{pO}_{2}$ between capillaries and assessment of the heterogeneity of the $\mathrm{pO}_{2}$ distributions were the key motivating factors for this study.

Oxygen-dependent quenching of phosphorescence ${ }^{16,17}$ has been used in the past to map oxygen distributions in subcutaneous tumors in animal models ${ }^{18-21}$ both macroscopically and microscopically, depending upon the magnification of the imaging method used. These measurements have revealed correlations between tumor $\mathrm{pO}_{2}$ levels and radiation sensitivity ${ }^{22,23}$. However, truly high-resolution depth-resolved imaging by phosphorescence is possible only at relatively shallow depths, not exceeding $0.5-0.7 \mathrm{~mm}$, by means of two-photon microscopy ${ }^{24-27}$, while volumetric phosphorescence lifetime tomography ${ }^{28}$ suffers from low resolution similar to other diffuse tomographic methods.

Recently, a new approach has been developed that combines phosphorescence quenching with excitation by the Cherenkov light generated within tissues that are subjected to high-energy radiation during fractionated radiotherapy ${ }^{29-31}$. This method, termed Cherenkov excited luminescence imaging (CELI), is used in the present study with the goal to directly image tumor $\mathrm{pO}_{2}$ distributions precisely at the time of radiation delivery. It is well known that hypoxic changes can cycle on the timescale of minutes, which is the typical time of a single radiation dose fraction ${ }^{32-34}$. Thus, the ability to image intratumoral $\mathrm{pO}_{2}$ at the actual time of the radiation delivery can be highly informative for optimization of clinical radiation therapy. In broad-beam CELI luminescence can be imaged that originates from the axial depths of up to 3-6 mm within the tumor, while the lateral spatial resolution may be as high as several hundred microns, depending on the luminescent source depth ${ }^{31}$. These features make CELI intrinsically capable of much higher depth resolution than alloptical tomographic imaging methods.

In this study, time-resolved CELI in combination with the phosphorescent probe Oxyphor PtG4 (Supplementary Fig. 1) is used to track oxygen changes in mouse xenograft tumors. Oxyphor PtG4 belongs to the family of dendritic oxygen probes ${ }^{21,35}$.
Its calibration parameters are stable in biological environments, and its phosphorescence lifetime reports selectively on absolute $\mathrm{pO}_{2}$ levels in tissues. Cherenkov light generated within the tumors, subjected to pulsed therapeutic megavolt (MV) X-ray irradiation, served as an internal light source to excite the probe's phosphorescence. A time-gated intensified charge coupled device (ICCD) camera, synchronized with the excitation pulses, captures emission at different delay times after each pulse (Supplementary Fig. 2a). The images of phosphorescence at different delays are used to construct an image of phosphorescence lifetimes by applying pixel-wise exponential fitting (Supplementary Fig. 2b). Notably, the tissue $\mathrm{pO}_{2}$ values are obtained from the phosphorescence lifetimes, as opposed to intensities, therefore being unaffected by optical heterogeneities of the medium and insensitive to the non-uniformity of the probe distribution. The practical usefulness of our imaging approach is evaluated by combining it with the hypofractioned radiation therapy scheme, whereby a single intravascular injection of Oxyphor PtG4 allows us to track oxygen dynamics in tumors during the course of the entire treatment.

\section{Results}

Bio-distribution of Oxyphor PtG4. To evaluate the biodistribution of Oxyphor PtG4 its phosphorescence was imaged ex vivo at different time points using a standard wide-field optical imaging system (IVIS 200 Spectrum CT, Perkin-Elmer, USA) after intravenous (IV) injection into the tail vein. The phosphorescence of Oxyphor PtG4 reached its peak within 5 min in the heart, lung, liver, spleen, kidneys and brain after the injection, while the highest signal in the tumor was detected after $24 \mathrm{~h}$ (Fig. 1a). The ex vivo images of the phosphorescence in individual organs at the $24 \mathrm{~h}$ time point are shown in Fig. $1 \mathrm{~b}$ along with the quantitative image analysis (Fig. 1c). Consistent with the ex vivo results, the images obtained in vivo (Fig. 1d), also at the $24 \mathrm{~h}$ time point, show that the phosphorescence from the tumors is $\sim 2$ times brighter than that from the normal tissue (Fig. 1e).

Long-term retention of Oxyphor PtG4 in tumors. Four mice were imaged using the IVIS instrument as well as by CELI every day for 5 days after IV injection of Oxyphor PtG4. The radiation for CELI was delivered using a beam of a large diameter, such that the images encompassed the entire abdomen area, including both tumors, as shown in the images of the excitation Cherenkov light (Fig. 2b). The phosphorescence images acquired using either IVIS or CELI indicated that sufficient amounts of Oxyphor PtG4 lasted in the tumor for at least 5 days, which provided an opportunity to image oxygenation in these tumors during multiple-fraction radiotherapy upon a single Oxyphor PtG4 injection (Fig. 2a, c). The phosphorescence intensity of Oxyphor PtG4 in the tumor and the normal tissue decreased monotonously from day 1 to day 5 (Fig. 2d, e). Since the signal intensity both in the normal tissue and tumor decreased gradually during the 5 days, the ratio "tumor signal:normal tissue signal" showed no significant decline from day 1 to day 5 (Fig. $2 \mathrm{f}$ ). Notably, the overall intensity of the signals measured using optical excitation was much higher than that in CELI, because the intensity of the light used for excitation in the IVIS instrument is much higher than that of the Cherenkov light. However, the Cherenkov radiation is generated within the tissue, and therefore the excitation has virtually no depth limitation, while the external optical excitation attenuates exponentially with depth (Supplementary Fig. 3a, b). As a result, CELI is capable of sampling oxygenation in much deeper tissue layers than all-optical phosphorescence imaging (Supplementary Fig. 3c, d). 
a
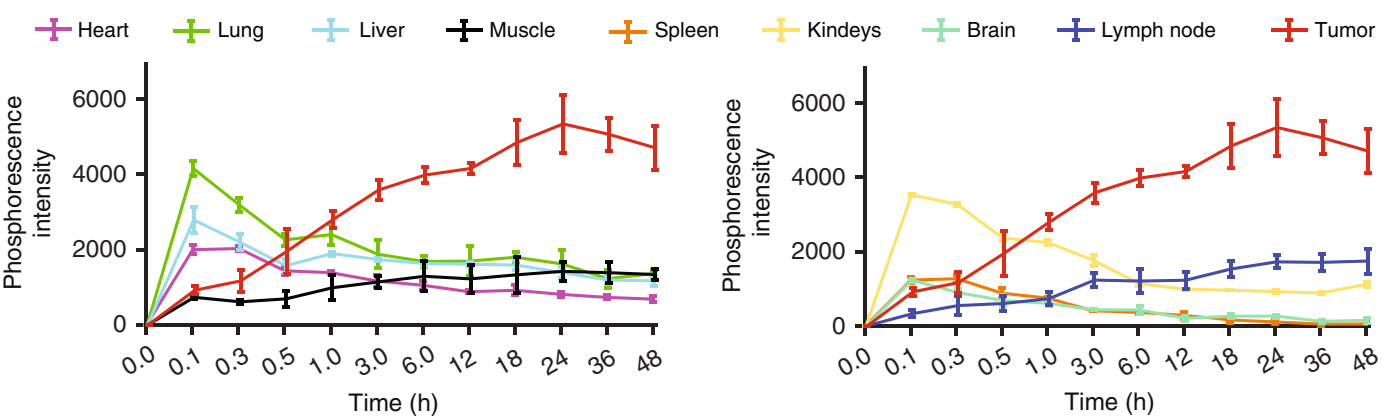

b

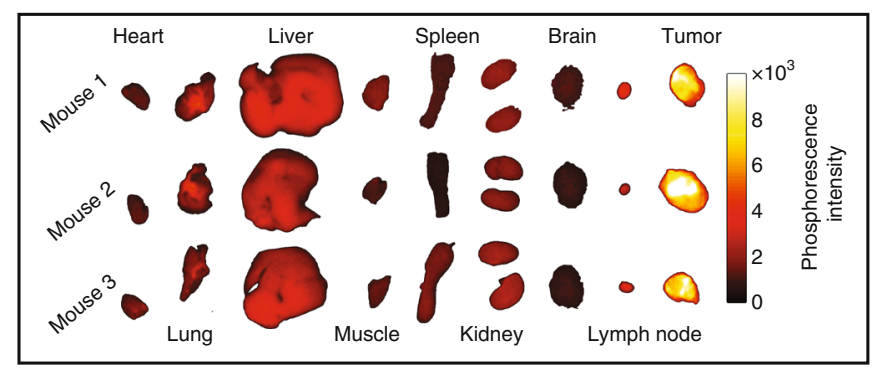

C
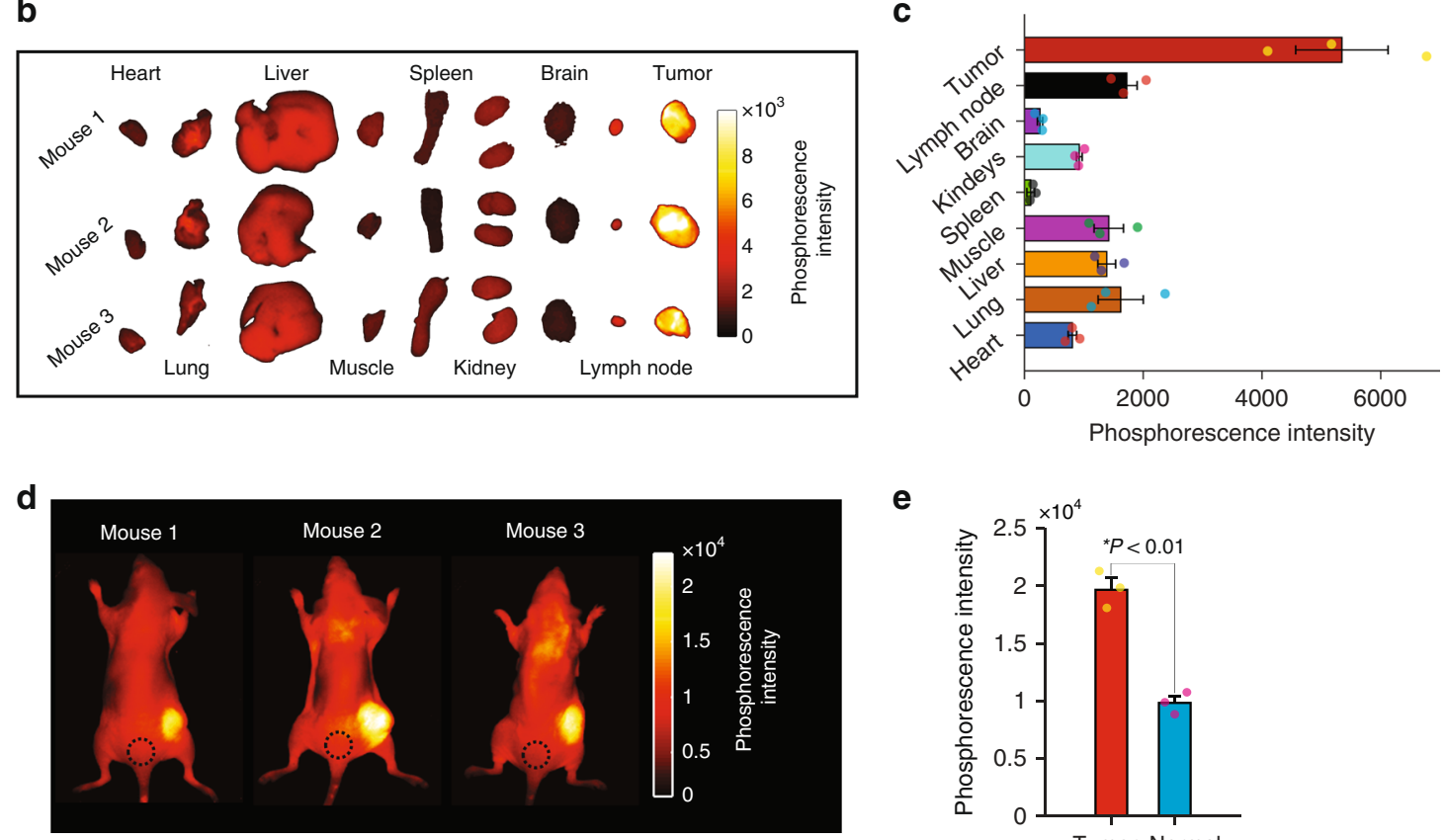

e

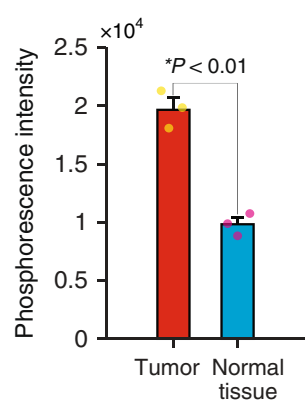

Fig. 1 Bio-distribution of Oxyphor PtG4 measured with IVIS instrument at different time points. a Phosphorescence from different organs and tumors $(n=3)$ measured at 12 time points after IV injection of Oxyphor PtG4 ( $n=3$ mice per time point, $n=36$ mice total). Ex vivo images of the phosphorescence from the excised organs (b) and their integrated intensities (c) at the $24 \mathrm{~h}$ time point after IV injection of Oxyphor PtG4 ( $n=3$ ). In vivo images of the phosphorescence (d) and the phosphorescence intensities in the tumors and regions of the normal tissue (marked by a black-dashed circle) (e) at $24 \mathrm{~h}$ after injection of Oxyphor PtG4. The mean signal from the tumors $\left(1.97 \pm 0.16 \times 10^{4}\right.$ counts, mean $\left.\pm \mathrm{SE}\right)$ was twice as high as that from the normal tissue $\left(0.98 \pm 0.09 \times 10^{4}\right.$ counts, mean $\left.\pm \mathrm{SE}\right)(n=3)$. Error bars represent standard error of the mean. Significant difference was analyzed by two-sample $t$-test.

In vivo $\mathrm{pO}_{2}$ imaging after local injection of Oxyphor PtG4. To demonstrate the ability to image $\mathrm{pO}_{2}$ in vivo by CELI in healthy (muscle) and tumor tissues pre- and post-euthanasia, Oxyphor PtG4 was locally injected into the tumor and into a thigh muscle. CELI was performed in four mice, first before (Fig. 3a) and then $30 \mathrm{~min}$ after (Fig. 3b) euthanasia. The data processing followed the standard protocol for wide-field phosphorescence lifetime imaging $^{21}$. The accuracy of the lifetime measurements by CELI was verified independently by performing ex vivo measurements using standard lifetime measurement instrumentation (Supplementary Fig. 4). The $\mathrm{pO}_{2}$ images (Fig. 3c, d) were calculated from the lifetime images using the Stern-Volmer equation and the calibration constants obtained in independent measurements. In live animals the $\mathrm{pO}_{2}$ values in the tumors were significantly lower than in the muscle tissue (Fig. 3c, e). As expected, the $\mathrm{pO}_{2}$ values both in the tumors and muscle rapidly dropped to near zero when mice were euthanatized (Fig. 3d, f). There was a significant variation between the median $\mathrm{pO}_{2}$ values for the tumor and muscle tissues in the four mice before euthanasia, but after euthanasia the $\mathrm{pO}_{2}$ was uniformly near zero in all animals (Fig. 3g).

In vivo $\mathrm{pO}_{2}$ imaging after IV injection of Oxyphor PtG4. Three mice were IV injected with Oxyphor PtG4 $(200 \mu \mathrm{L}$ of $200 \mu \mathrm{M}$ solution) through the tail vein, so that the final concentration of the probe in the blood was $\sim 20 \mu \mathrm{M}$, and CELI was performed $24 \mathrm{~h}$ later (Fig. 4a). The $\mathrm{pO}_{2}$ images (Fig. $4 \mathrm{~b}$ ) and their histograms (Fig. 4c) revealed a significant degree of hypoxia in the tumors versus the surrounding tissue. Statistically significant differences $(P<0.005, n=3$, two-sample $t$-test) were observed between the median $\mathrm{pO}_{2}$ values (Fig. $\left.4 \mathrm{~d}\right)$ in the tumor $(11.3 \pm 0.8$ Torr, mean $\pm \mathrm{SE}, n=3)$ and normal tissue $(20.4 \pm 1.1$ Torr, mean \pm SE, $n=$ 3). The $\mathrm{pO}_{2}$ distributions in the tumor regions were found to be highly heterogeneous (Fig. 4e). Analysis of locally high and low $\mathrm{pO}_{2}$ sub-regions revealed that they are characterized by significantly different $\mathrm{pO}_{2}$ histograms (Fig. 4f). An arbitrary threshold of 10 Torr was applied to divide the $\mathrm{pO}_{2}$ image into 
a

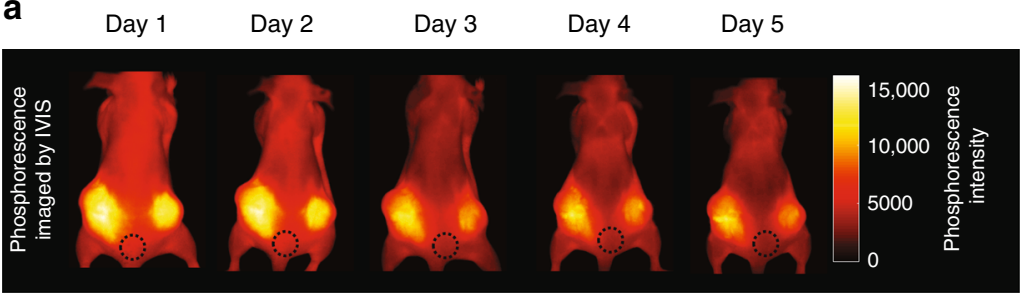

b

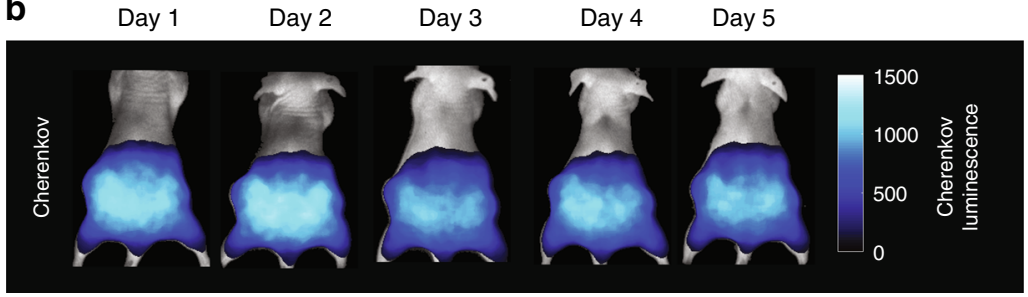

C

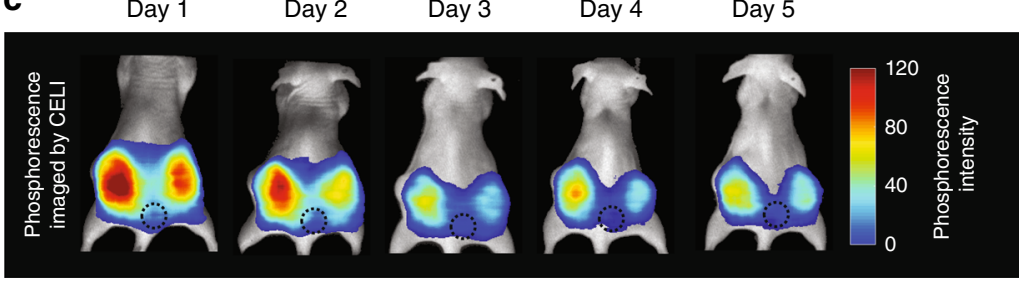

d

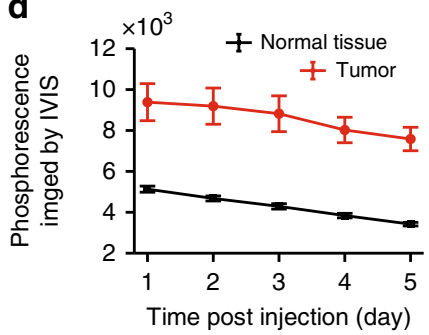

e

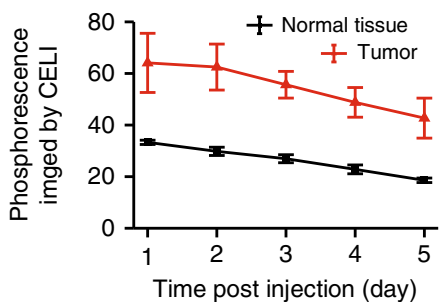

$\mathbf{f}$

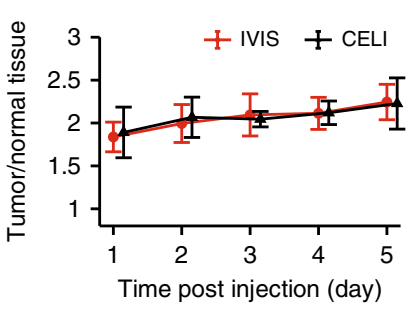

Fig. 2 Longitudinal in vivo imaging of phosphorescence during $\mathbf{5}$ days after a single IV injection of Oxyphor PtG4. a Phosphorescence intensity images measured using wide-field optical excitation in a standard IVIS instrument. b Integrated intensity images of the Cherenkov light along with the phosphorescence of Oxyphor PtG4, acquired during the time of the radiation delivery. c Phosphorescence intensity images by CELI. Average intensity of the phosphorescence acquired by IVIS (d) and CELI (e) for the tumor area and for the normal tissue $(n=4)$. $\mathbf{f}$ The ratio of the signals tumor:normal tissue as imaged by IVIS and CELI $(n=4)$. Error bars represent standard error of the mean.

hypoxic ( $\mathrm{pO}_{2}<10$ Torr) and normoxic $\left(\mathrm{pO}_{2} \geq 10\right.$ Torr) regions. According to this division, there were two hypoxic regions in the tumor (Fig. 4g), which accounted for $5 \%$ of the imaged area (Fig. 4h). These regions were smaller than $1 \mathrm{~mm}$ in diameter and were separated by a distance of $0.4 \mathrm{~mm}$ (Fig. $4 \mathrm{e}$ ).

In vivo $\mathrm{pO}_{2}$ imaging during multiple-fraction radiotherapy. Six mice with subcutaneous MDA-MB-231 or FaDu tumors were imaged while undergoing 5 days-long multiple-fraction radiotherapy. The radiation was delivered using a $6 \mathrm{MV} \mathrm{X}$-ray beam with $5 \mathrm{~Gy} /$ fraction daily, with the total radiation dose of $25 \mathrm{~Gy}$. Oxyphor PtG4 was IV injected $24 \mathrm{~h}$ before the first treatment, and CELI was performed during each fraction of radiation. The $\mathrm{pO}_{2}$ distributions in both MDA-MB-231 and FaDu tumors revealed a high degree of heterogeneity 36,37 . Importantly, the hypoxic regions decreased in size from one fraction to the next, and the decrease was much more pronounced in the case of the MDAMB-231 tumors (Fig. 5a). In both tumor types, the $\mathrm{pO}_{2}$ values were below 20 Torr on the first day, and they increased up to $\sim 30$ Torr for MDA-MB-231 and up 25 Torr for $\mathrm{FaDu}$ tumors (Fig. 5b). The median $\mathrm{pO}_{2}$ values in the MDA-MB-231 tumors increased markedly as the radiotherapy progressed, while no obvious changes were seen in the case of FaDu tumors (Fig. 5c), implying that more hypoxic and radio-resistant tumors allowed for less local control, which presumably resulted in a higher rate of their survival ${ }^{38}$ (Fig. $5 \mathrm{~d}$ ). The response of the tumor volume was delayed relative to the response of the local $\mathrm{pO}_{2}$, i.e. MDAMB-231 tumors started to decrease in size on day 5 and $\mathrm{FaDu}$ tumors only on day 9 from the start of the therapy (Fig. 5e).
Overall, the changes in the $\mathrm{pO}_{2}$ distributions and the tumor volumes suggest that MDA-MB-231 tumors are more prone to the radiation damage compared to FaDu tumors (see Supplementary Fig. 5 for additional results and Supplementary Fig. 6 for histological evaluation of the tumor tissue after radiation).

\section{Discussion}

There is a need to measure $\mathrm{pO}_{2}$ distributions in tumors with high spatial resolution ${ }^{39-41}$ in order to identify hypoxic fractions, which are known to be less sensitive to radiation. CELI in combination with Oxyphor PtG4 may help to identify such hypoxic regions and thus help navigating and optimizing clinical radiation therapy. The fact that CELI can be performed simultaneously with fractionated therapy makes it particularly clinically relevant. In this version of CELI the limiting factor is the optical perspective of view, i.e. clear optical access to the tumor is required. In addition, the method is capable of sensing $\mathrm{pO}_{2}$ only in a few millimeters thick layer of subcutaneous tissue. However, future realizations of the technique could be adapted for imaging of intracavitary or near-surface tumors. Most importantly, while this imaging method presently can be utilized only in pre-clinical studies, it is uniquely capable of non-invasive sampling of $\mathrm{pO}_{2}$ across tumors with submillimeter spatial resolution exactly at the time of the radiotherapy delivery.

In this work we showed that Oxyphor PtG4 accumulates preferentially within tumors and remains there for at least 5 days. This localization is likely due to the enhanced permeability and retention (EPR) effect. Localization studies (Supplementary Fig. 7) revealed that the probe distributes across the entire 
a

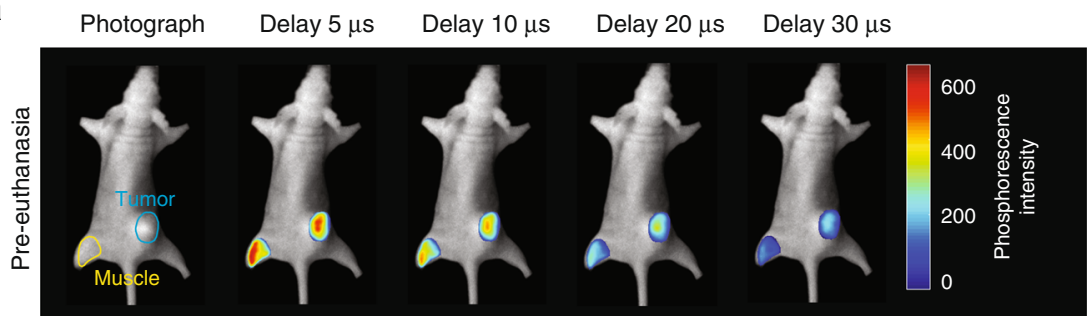

C

b
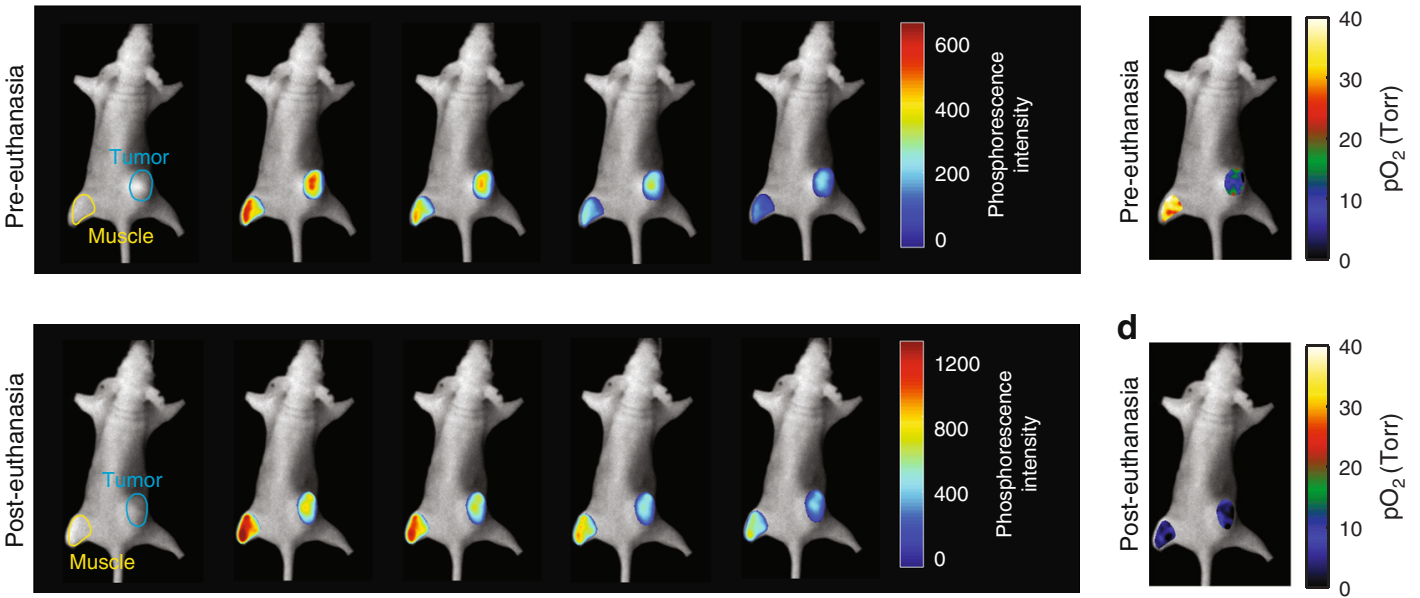

e
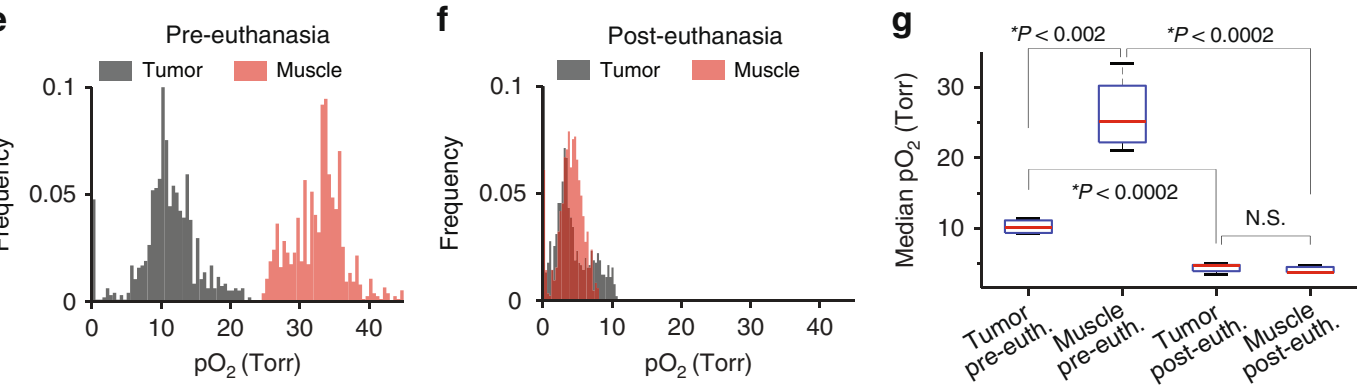

Fig. 3 CELI of oxygen in tumors and muscle in vivo before and $\mathbf{3 0}$ min after euthanasia. Oxyphor PtG4 (50 $\mu \mathrm{L}$ of $200 \mu \mathrm{M}$ solution) was locally injected into the MDA-MB-231 tumor (blue circle) and into muscle (yellow circle) before the imaging session. $\mathbf{a}, \mathbf{b}$ Phosphorescence intensity images acquired at different delays relative to the radiation pulse before (a) and after euthanasia (b). The phosphorescence images are overlaid on a photograph of the mouse, which was taken before CELI. The tumor and muscle areas used for the analysis are encircled. Tissue oxygen maps $(\mathbf{c}, \mathbf{d})$ and $\mathrm{pO}_{2}$ histograms $(\mathbf{e}, \mathbf{f})$ before and after euthanasia. $\mathbf{g}$ Average levels of oxygen in the tumor and muscle before and after euthanasia $(n=4)$. Boxplot shows median and interquartile range; whiskers indicate the range. Statistics was performed using two-sample $t$-test.

interstitial space of the tumor. There may be slightly higher sequestration into necrotic areas at later time points, but there is also more necrosis at these time points, making this further sequestration pattern hard to assay with certainty. Importantly, the probe is present in the tumors throughout the entire time course of the studies, and stronger quenching of the phosphorescence at higher oxygen concentrations, characteristic of normal tissues, results in the phosphorescence from hypoxic tumors being significantly brighter (Fig. 1e), providing a natural signal selection mechanism.

A single injection of Oxyphor PtG4 allowed $\mathrm{pO}_{2}$ imaging over multiple days during fractionated radiotherapy. Since $\mathrm{pO}_{2}$ is estimated from the phosphorescence lifetime, the decrease in the concentration of the probe due to its clearance from the body does not affect the ability to quantify oxygen, as long as enough probe is present for acquiring adequate signals. Longer-term residence of Oxyphor PtG4 remains to be investigated as well as potential long-term toxicity issues; however, cell metabolic activity measurements using a standard MTT assay showed that the cell viability was above $90 \%$ when the cells were incubated with Oxyphor PtG4 at the concentration of $40 \mu \mathrm{M}$ for $24 \mathrm{~h}$ (Supplementary Fig. 8), that is two times higher than in all in vivo experiments performed in this study. A similar result has been obtained previously in the toxicity tests of the probe Oxyphor $\mathrm{PdG}_{4}{ }^{42}$, which is structurally almost identical to Oxyphor PtG4. Hematoxylin and eosin staining assay did not show differences between tissues of the main organs of animals infused with
Oxyphor PtG4 vs phosphate buffer saline (PBS) (Supplementary Fig. 9). Likewise, blood chemistry tests (Supplementary Table S1) revealed no difference between treatments with Oxyphor PtG4 vs PBS. Overall, our results suggest that Oxyphor PtG4 has no toxic effects on the physiological function of cells, and its clearance from the body occurs presumably by the renal process.

The excitation source in CELI is the Cherenkov light generated along the pathways of MV X-ray beams. Therefore, excitation occurs at all depths where the radiation dose is deposited, including deep within tissues. Nevertheless, the detection of the phosphorescence emission is still limited by the tissue absorption and scattering. Considering the limitations of imaging sensors and the moderate brightness of the partially quenched phosphorescence, the maximal imaging depth is currently estimated to be $\sim 2 \mathrm{~cm}$ (Supplementary Fig. 3). The depth can be increased by employing brighter phosphorescent probes, such as recently developed Oxyphor $2 \mathrm{P}^{43}$ and/or using cameras with sensitivity optimized specifically for the phosphorescence spectral range (750-850 nm). The scope of potential applications of CELI then can be extended on imaging of near-surface tumors, such as head and neck tumors, as well as on pre-clinical assessments of oxygenation during therapy in laboratory animals. It is also possible that intracavity measurements could be achieved with appropriate catheter-based cameras.

This study was specifically designed to use clinically relevant stereotactic hypofractionated radiotherapy doses and 6MV X-rays from a clinical LINAC system. In our recent work we 

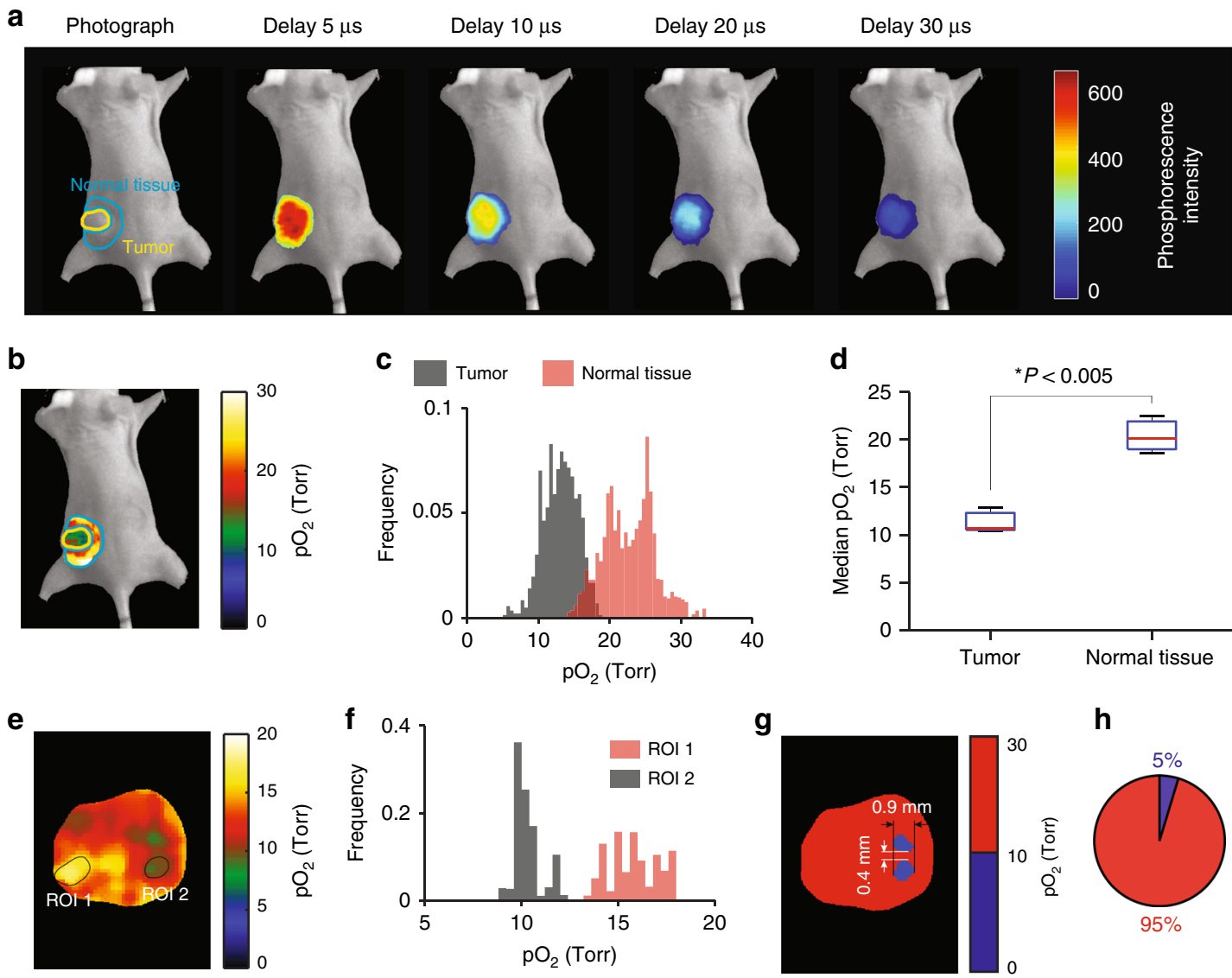

h

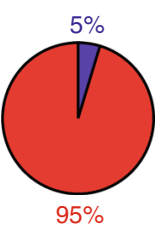

Fig. $\mathbf{4}$ In vivo imaging of $\mathbf{p O}_{\mathbf{2}}$ in mice $\mathbf{2 4} \mathbf{~ h}$ after IV injection of Oxyphor PtG4. a Phosphorescence intensity images acquired at different delays of 5 , 10, 20 , and $30 \mu$ s relative to the excitation pulse, overlaid with a photograph of the mouse. The regions corresponding to the tumor and the normal surrounding tissue are shown by the yellow and blue circles in the first image. $\mathbf{b}$ Tissue $\mathrm{pO}_{2}$ map. $\mathbf{c} \mathrm{pO}_{2}$ histograms. $\mathbf{d}$ Median $\mathrm{pO}_{2}$ values in the tumor and surrounding tissue $(n=3)$. Boxplot shows median and interquartile range; whiskers indicate the range. Statistics was performed using two-sample $t$-test. e Enlarged view of the tumor region in the $\mathrm{pO}_{2}$ image. $\mathbf{f} \mathrm{pO}_{2}$ histograms of sub-regions (ROIs) outlined by black curves in $\mathbf{e}$. $\mathbf{g}$ Areas shown in blue were characterized by $\mathrm{pO}_{2}<10$ Torr. $\mathbf{h}$ Fraction of the tumor having $\mathrm{pO}_{2}<10$ Torr.

demonstrated the possibility of high-resolution CELI during standard clinical intensity-modulated radiotherapy in a wholebreast geometry. We do not anticipate technical difficulties in adapting the latter method for fractionated treatment ${ }^{44}$.

Compared to the current clinical $\mathrm{pO}_{2}$ measurement modalities, the CELI approach is capable of significantly higher spatial resolution and allows image acquisition simultaneously with radiotherapy delivered in multiple fractions. One current limitation of the technique is that only two-dimensional $\mathrm{pO}_{2}$ images can be obtained. Our previous studies have shown that threedimensional (3D) tomographic imaging of phosphorescence using light sheet excitation and diffuse optical tomography image reconstruction methods makes it possible to obtain $3 \mathrm{D} \mathrm{pO}_{2}$ maps with submillimeter resolution in vivo ${ }^{29}$. However, the tomography technique relies on thin sheet-like X-ray beams, which are not routinely used in the current clinical practice. Furthermore, scanning of the entire tissue volume for tomographic reconstructions may be difficult to incorporate into protocols of clinical therapy. In contrast, the simplified method reported here can be easily added to clinical protocols, enabling evaluation of tumor $\mathrm{pO}_{2}$ histograms at the time of the radiation dose delivery, which is a long-sought goal in tumor therapy.

\section{Methods}

Ethics statement. Experimental procedures involving live animals were carried out in accordance with the protocols approved by Dartmouth Institutional Animal Care and Use Committee (Protocol Numbers 00002173). Subcutaneous and intravenous injections were performed under anesthesia that was induced and maintained with isoflurane. All efforts were made to minimize animal suffering.

Oxygen probe Oxyphor PtG4. Oxyphor PtG4 belongs to the family of dendritic oxygen probes ${ }^{35}$. It is a direct analog of the previously published probe Oxyphor $\mathrm{PdG}_{4}{ }^{21}$, different only by the metal in the porphyrin: platinum vs palladium (Supplementary Fig. 1a). The porphyrin is responsible for the key optical properties of the probe, which include two intense absorption bands $\left(\lambda_{\max }=435\right.$ and $\left.624 \mathrm{~nm}\right)$ and strong phosphorescence $\left(\lambda_{\max }=780 \mathrm{~nm}, \mathrm{QY} \sim 0.07\right.$ in deoxygenated aq. solutions) occurring in the tissue near-infrared window (Supplementary Fig. 1b). The porphyrin molecule is encapsulated in a hydrophobic jacket, formed by a folded aryl-glycine dendrimer. The dendrimer protects the porphyrin from interactions with endogenous macromolecules and attenuates the rate of oxygen diffusion in the vicinity of the porphyrin, bringing the probe's sensitivity into the desired $\mathrm{pO}_{2}$ range. The exterior of the probe is modified with multiple polyethyleneglycol (PEG) residues, making it hydrophilic and inert with respect to the endogenous macromolecules. Due to the extensive PEGylation the probe's calibration parameters remain unaltered in biological environments. The molecular weight of Oxyphor PtG4 is $\sim 35 \mathrm{kDa}$, and the approximate diameter of the molecule in an aqueous solution is $\sim 6 \mathrm{~nm}$. Therefore, Oxyphor PtG4 can easily diffuse into the tumor interstitial space from the leaky vasculature: a phenomenon known as EPR effect. However, the same PEG coating prevents Oxyphor PtG4 from permeating cellular membranes. Thus, measurements using Oxyphor PtG4 give average intratumoral extravascular $\mathrm{pO}_{2}$.

The phosphorescence lifetime of Oxyphor PtG4 at $37^{\circ} \mathrm{C}$ under fully anoxic conditions is $\tau_{0}=45 \mu \mathrm{s}$. The response of the phosphorescence to oxygen was calibrated by simultaneously measuring the probe's phosphorescence lifetime and $\mathrm{pO}_{2}$ in solution using a high-precision oxygen electrode, while gradually decreasing oxygen concentration by substituting oxygen with argon ${ }^{21}$. The Stern-Volmer oxygen quenching plot was linear throughout the physiological $\mathrm{pO}_{2}$ range $(0-150$ Torr) with the quenching constant $k_{\mathrm{q}}=366 \mathrm{Torr}^{-1} \mathrm{~s}^{-1}$. In addition, the probe was calibrated using Cherenkov excitation by LINAC pulses and four acquisition 
a
Day 1
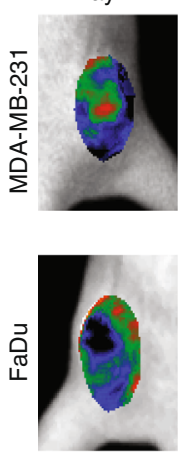

Day 2
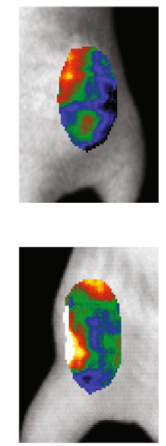

Day 3
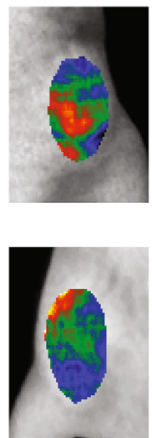

Day 4
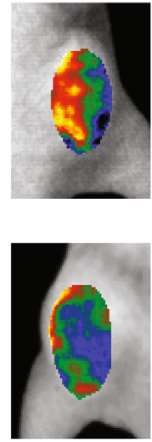

Day 5
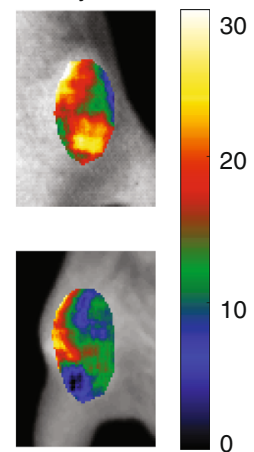

30

20 c
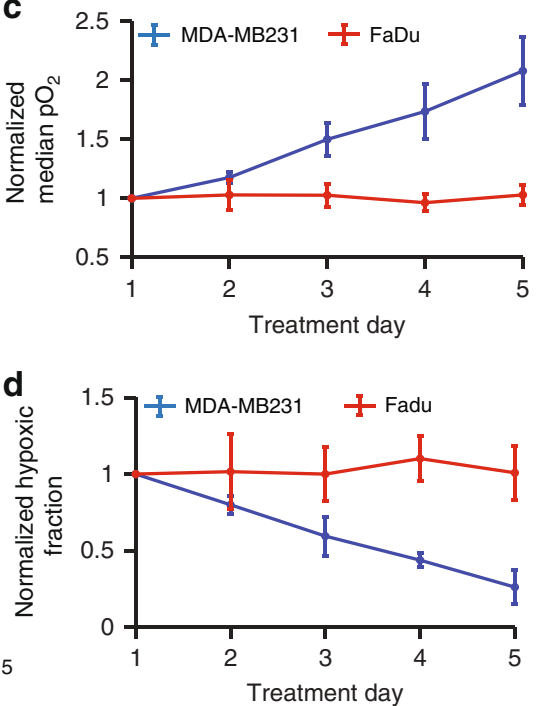

b
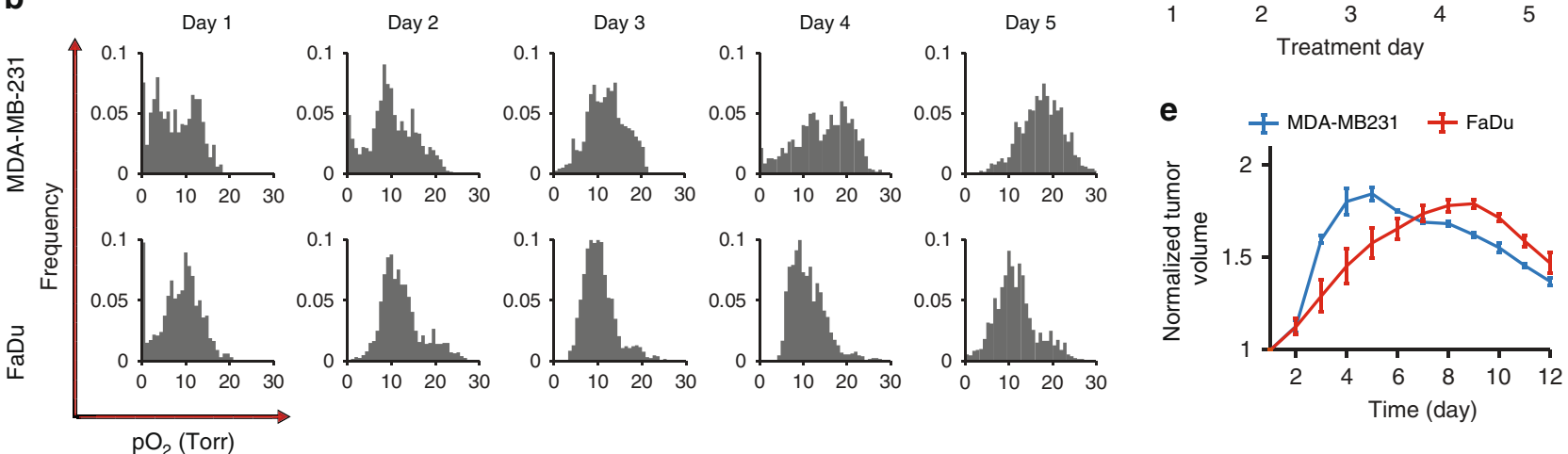

Fig. $\mathbf{5}$ In vivo longitudinal $\mathbf{p O}_{\mathbf{2}}$ imaging of mice with two tumor lines during $\mathbf{5}$ days-long fractionated radiotherapy. $\mathbf{a}, \mathbf{b}$ Examples of $\mathrm{pO}_{2}$ images (a) and of histograms (b) acquired during each day of the radiotherapy treatment. c, d Median $\mathrm{pO}_{2}$ changes (c) and hypoxic fraction changes (d). Hypoxic fraction is defined here as the ratio of the area with $\mathrm{pO}_{2}<10$ Torr to the total tumor area. The changes in the median $\mathrm{pO}_{2}$ (c) and in the hypoxic fraction (d) are shown relative to the respective values on the first treatment day. e Relative tumor volume changes during and after the radiotherapy, normalized by the volume prior to the treatment. All data are shown as mean \pm standard error of the mean $(n=3$ in $\mathbf{c}, \mathbf{d}, \mathbf{e})$.

windows with delays of $5,10,20$, and $30 \mu$ s. The signal integration time after each delay was $200 \mu \mathrm{s}$, and 360 windows were accumulated for each delay. This latter calibration was performed using an air-equilibrated solution of Oxyphor PtG4 $\left(\mathrm{pO}_{2}=150\right.$ Torr at $\left.37^{\circ} \mathrm{C}\right)$ and a fully deoxygenated solution $\left(\mathrm{pO}_{2}=0\right.$ Torr at $37^{\circ} \mathrm{C}$ ) (Supplementary Fig. 1c).

Tumor cell lines. The human breast cancer cell line MDA-MB-231 was purchased from Perkin-Elmer/Caliper (Cat. No.: 119369), and human head neck cancer cell line FaDu was purchased directly from American Type Culture Collection (ATCC HTB-43, Manassas Virginia). These are not listed in the ICLAC database of crosscontaminated or misidentified cell lines. Cells were grown in culture media in a humidified incubator at $37^{\circ} \mathrm{C}$ and $5 \% \mathrm{CO}_{2}$ in MEM with $10 \%(\mathrm{v} / \mathrm{v}$ ) fetal bovine serum (FBS), $100 \mathrm{U} / \mathrm{mL}$ penicillin, and $100 \mu \mathrm{g} / \mathrm{mL}$ streptomycin. When ready for use, the cells were trypsinized, counted, pelleted, and resuspended for injection.

Animal preparation. All procedures followed the protocol approved by the Dartmouth Institutional Animal Care and Use Committee. Nude female mice 6-8 weeks of age (Charles River Labs, Wilmington, MA) were involved in this study. The mice were housed in the Dartmouth central animal facility and fed special diet-MP biomedical purified diet. $10^{6}$ MDA-MB-231 or $10^{6} \mathrm{FaDu}$ tumor cells were injected subcutaneously under the skin on the flank of each mouse. After approximately 2 weeks of growth, animals were chosen for imaging when their tumor diameter reached approximately $8 \mathrm{~mm}$ in size. On the day of the initial use, mice were anesthetized, and Oxyphor PtG4 was either IV injected into a tail vein ( $200 \mu \mathrm{L}$ of $200 \mu \mathrm{M}$ solution) or locally injected into the tumor or normal muscle ( $50 \mu \mathrm{L}$ of $200 \mu \mathrm{M}$ solution), right before the start of the first in vivo imaging session.

In vitro imaging of bio-distributions of Oxyphor PtG4. Wide-field optical imaging (IVIS 200 Spectrum CT; Perkin-Elmer, USA) was used to quantify phosphorescence from harvested organs (heart, lung, liver, spleen, kidneys, brain, muscle, lymph node, and tumor). The excitation and emission wavelengths were 640 and $780 \mathrm{~nm}$, respectively. The integration time was $0.2 \mathrm{~s}$ with binning of 2 . In total, 36 mice with MDA-MB-231 tumors were IV injected with Oxyphor PtG4 ( $100 \mu \mathrm{L}$ of $100 \mu \mathrm{M}$ solution) through the tail vein. At each of the 12 time points of $0 \mathrm{~min}, 5 \mathrm{~min}, 15 \mathrm{~min}, 30 \mathrm{~min}, 1 \mathrm{~h}, 3 \mathrm{~h}, 6 \mathrm{~h}, 12 \mathrm{~h}, 18 \mathrm{~h}, 24 \mathrm{~h}, 36 \mathrm{~h}$, and $48 \mathrm{~h}$, organs from three mice were imaged.

CELI during radiotherapy. Cherenkov light was induced in the tissue by irradiation from a linear accelerator (Varian Linac 2100CD; Varian Medical System) at the Norris Cotton Cancer Center, Dartmouth-Hitchcock Medical Center. The beam size was adjusted to cover the entire tumor area. To minimize the background, all lights in the room were switched off. The imaging system consisted of a time-gated intensified CCD camera (ICCD, PI-MAX4 1024i; Princeton Instruments), a lens (Canon EF $135 \mathrm{~mm}$ f/2L USM), and a $750 \mathrm{~nm} / 100 \mathrm{~nm}$ band pass filter. The camera was contained in a homemade lead box, placed $\sim 2 \mathrm{~m}$ away from the imaging field to protect it from Compton scattered X-ray photons. The noise induced by X-ray photons was therefore significantly suppressed, and the signal-tonoise ratio (SNR) was more than seven times higher after shielding (Supplementary Fig. 10). The time-gated ICCD camera was synchronized with the radiation pulses, which were $\sim 3.25 \mu$ s long and delivered with $360 \mathrm{~Hz}$ repetition rate. The camera gate was turned on at $5,10,20$, and $30 \mu$ s delay times and the phosphorescence was integrated on the CCD for $200 \mu$ s. The intensifier gain was $\times 100$.

Longitudinal imaging study. The mice injected with Oxyphor PtG4 were continuously imaged by CELI for 5 days during their hypofractionated radiotherapy, and also imaged with the IVIS instrument for comparative purposes. All mice were under general anesthesia (isofluorane $1-3 \%$ admixed to $\mathrm{O}_{2}$ and delivered through a nose cone) throughout imaging.

Cytotoxicity assay. MTT assays were performed on MDA-MB-231 and Fadu cells. Solutions of Oxyphor PtG4 at different concentrations $(0,10,20$, and $40 \mu \mathrm{M}$ 
diluted in PBS) were added to the wells. The samples were incubated for $24 \mathrm{~h}$ in a cell culture incubator, and $10 \mu \mathrm{L}$ of $5 \mathrm{mg} / \mathrm{mL}$ MTT solution [3-(4,5-dimethylthiazolyl-2)-2,5-diphenyl tetrazolium bromide; MP Biomedical, Solon OH] was added to each well. After incubating the plate for another $6 \mathrm{~h}$, the media was removed from all wells and $150 \mu \mathrm{L}$ of acidified isopropanol was added to each well. Finally, the percentage of living cells was determined based on the measured optical density (OD) at $540 \mathrm{~nm}$.

Statistical analysis. All statistical analyses for differences in $\mathrm{pO}_{2}$ between treatment groups were done using two-sample $t$-test with Matlab (Mathworks, Inc., Natick, MA).

Reporting summary. Further information on research design is available in the Nature Research Reporting Summary linked to this article.

\section{Data availability}

The data that support the findings of this study are available within the paper and its Supplementary Information. Source datasets generated and analyzed during the study are available from the corresponding author upon request.

\section{Code availability}

MATLAB routines for this study are available from the corresponding author upon request.

Received: 31 July 2019; Accepted: 4 January 2020;

Published online: 29 January 2020

\section{References}

1. Oronsky, B. T., Knox, S. J. \& Scicinski, J. Six degrees of separation: the oxygen effect in the development of radiosensitizers. Transl. Oncol. 4, 189-198 (2011).

2. Shannon, A. M., Bouchier-Hayes, D. J., Condron, C. M. \& Toomey, D. Tumour hypoxia, chemotherapeutic resistance and hypoxia-related therapies. Cancer Treat. Rev. 29, 297-307 (2003).

3. Becker, A. et al. Oxygenation of squamous cell carcinoma of the head and neck: comparison of primary tumors, neck node metastases, and normal tissue. Int J. Radiat. Oncol. Biol. Phys. 42, 35-41 (1998).

4. Horsman, M. R., Nordsmark, M. \& Overgaard, J. Techniques to assess the oxygenation of human tumors. State of the art. Strahlenther. Onkol. 174, 2-5 (1998).

5. Nordsmark, M. et al. Hypoxia in human soft tissue sarcomas: adverse impact on survival and no association with p53 mutations. Br. J. Cancer 84, 1070-1075 (2001).

6. Overgaard, J. Hypoxic modification of radiotherapy in squamous cell carcinoma of the head and neck-a systematic review and meta-analysis. Radiother. Oncol. 100, 22-32 (2011).

7. Hoskin, P. J., Rojas, A. M., Saunders, M. I., Bentzen, S. M. \& Motohashi, K. J. Carbogen and nicotinamide in locally advanced bladder cancer: early results of a phase-III randomized trial. Radiother. Oncol. 91, 120-125 (2009).

8. Gaertner, F. C., Souvatzoglou, M., Brix, G. \& Beer, A. J. Imaging of hypoxia using PET and MRI. Curr. Pharm. Biotechnol. 13, 552-570 (2012).

9. Nozue, M. et al. Interlaboratory variation in oxygen tension measurement by Eppendorf "Histograph" and comparison with hypoxic marker. J. Surg. Oncol. 66, 30-38 (1997).

10. Nordsmark, M. et al. The prognostic value of pimonidazole and tumour pO2 in human cervix carcinomas after radiation therapy: a prospective international multi-center study. Radiother. Oncol. 80, 123-131 (2006).

11. Hendrickson, K. et al. Hypoxia imaging with [F-18] FMISO-PET in head and neck cancer: potential for guiding intensity modulated radiation therapy in overcoming hypoxia-induced treatment resistance. Radiother. Oncol. 101, 369-375 (2011).

12. Lock, S. et al. Residual tumour hypoxia in head-and-neck cancer patients undergoing primary radiochemotherapy, final results of a prospective trial on repeat FMISO-PET imaging. Radiother. Oncol. 124, 533-540 (2017).

13. O'Connor, J. P. B., Robinson, S. P. \& Waterton, J. C. Imaging tumour hypoxia with oxygen-enhanced MRI and BOLD MRI. Br. J. Radiol. 92, 20180642 (2019).

14. Baudelet, C. \& Gallez, B. How does blood oxygen level-dependent (BOLD) contrast correlate with oxygen partial pressure (pO2) inside tumors? Magn Reson. Med. 48, 980-986 (2002).

15. Okunieff, P., Urano, M., Kallinowski, F., Vaupel, P. \& Neuringer, L. J. Tumors growing in irradiated tissue: oxygenation, metabolic state, and $\mathrm{pH}$. Int $J$. Radiat. Oncol. Biol. Phys. 21, 667-673 (1991).
16. Vanderkooi, J. M., Maniara, G., Green, T. J. \& Wilson, D. F. An optical method for measurement of dioxygen concentration based upon quenching of phosphorescence. J. Biol. Chem. 262, 5476-5482 (1987).

17. Rumsey, W. L., Vanderkooi, J. M., and Wilson, D. F. Imaging of phosphorescence: A novel method for measuring the distribution of oxygen in perfused tissue. Science 241, 1649-1651 (1988).

18. Wilson, D. F. \& Cerniglia, G. J. Localization of tumors and evaluation of their state of oxygenation by phosphorescence imaging. Cancer Res. 52, 3988-3993 (1992).

19. Vinogradov, S. A. et al. Noninvasive imaging of the distribution in oxygen in tissue in vivo using near-infrared phosphors. Biophys. J. 70, 1609-1617 (1996).

20. Palmer, G. M. et al. Optical imaging of tumor hypoxia dynamics. J. Biomed. Opt. 15, 066021 (2010).

21. Esipova, T. V. et al. Two new "protected" oxyphors for biological oximetry: properties and application in tumor imaging. Anal. Chem. 83, 8756-8765 (2011).

22. Cerniglia, G. J. et al. Intravascular oxygen distribution in subcutaneous 9L tumors and radiation sensitivity. J. Appl. Physiol. 82, 1939-1945 (1997).

23. Erickson, K. et al. Effect of longitudinal oxygen gradients on effectiveness of manipulation of tumor oxygenation. Cancer Res. 63, 4705-4712 (2003).

24. Finikova, O. S. et al. Oxygen microscopy by two-photon-excited phosphorescence. ChemPhysChem 9, 1673-1679 (2008).

25. Sakadžić, S. et al. Two-photon high-resolution measurement of partial pressure of oxygen in cerebral vasculature and tissue. Nat. Med. 7,755 (2010).

26. Lecoq, J. et al. Simultaneous two-photon imaging of oxygen and blood flow in deep cerebral vessels. Nat. Med. 17, 893 (2011).

27. Spencer, J. A. et al. Direct measurement of local oxygen concentration in the bone marrow of live animals. Nature 508, 269 (2014).

28. Apreleva, S. V., Wilson, D. F. \& Vinogradov, S. A. Tomographic imaging of oxygen by phosphorescence lifetime. Appl. Opt. 45, 8547-8559 (2006).

29. Pogue, B. W. et al. Maps of in vivo oxygen pressure with submillimetre resolution and nanomolar sensitivity enabled by Cherenkov-excited luminescence scanned imaging. Nat. Biomed. Eng. 2, 254-264 (2018).

30. Zhang, R. et al. Cherenkov-excited luminescence scanned imaging. Opt. Lett. 40, 827-830 (2015).

31. LaRochelle, E. P. M., Shell, J. R., Gunn, J. R., Davis, S. C. \& Pogue, B. W. Signal intensity analysis and optimization for in vivo imaging of Cherenkov and excited luminescence. Phys. Med. Biol. 63, 085019 (2018).

32. Brown, J. M. Evidence for acutely hypoxic cells in mouse tumours, and a possible mechanism of reoxygenation. Br. J. Radiol. 52, 650-656 (1979).

33. Chaplin, D. J., Olive, P. L. \& Durand, R. E. J. Cr Intermittent blood flow in a murine tumor: radiobiological effects. Cancer Res. 47, 597-601 (1987).

34. Trotter, M. J., Chaplin, D. J., Durand, R. E. \& Olive, P. L. The use of fluorescent probes to identify regions of transient perfusion in murine tumors. Int. J. Radiat. Oncol. Biol. Phys. 16, 931-934 (1989).

35. Lebedev, A. Y. et al. Dendritic phosphorescent probes for oxygen imaging in biological systems. ACS Appl. Mater. Interfaces 1, 1292-1304 (2009).

36. Dhani, N. et al. Analysis of the intra-and intertumoral heterogeneity of hypoxia in pancreatic cancer patients receiving the nitroimidazole tracer pimonidazole. Br. J. Cancer 113, 864 (2015).

37. Brurberg, K., Graff, B. \& Rofstad, E. Temporal heterogeneity in oxygen tension in human melanoma xenografts. Br. J. Cancer 89, 350 (2003).

38. Brizel, D. M., Sibley, G. S., Prosnitz, L. R., Scher, R. L. \& Dewhirst, M. W. Tumor hypoxia adversely affects the prognosis of carcinoma of the head and neck. Int J. Radiat. Oncol. Biol. Phys. 38, 285-289 (1997).

39. Lin, A. \& Hahn, S. M. Hypoxia imaging markers and applications for radiation treatment planning. Semin. Nucl. Med. 42, 343-352 (2012).

40. Horsman, M. R., Mortensen, L. S., Petersen, J. B., Busk, M. \& Overgaard, J. Imaging hypoxia to improve radiotherapy outcome. Nat. Rev. Clin. Oncol. 9, 674-687 (2012).

41. Horsman, M. R. \& Overgaard, J. The impact of hypoxia and its modification of the outcome of radiotherapy. J. Radiat. Res. 57, 90-98 (2016).

42. Ceroni, P. et al. Evaluation of phototoxicity of dendritic porphyrin-based phosphorescent oxygen probes: an in vitro study. Photochem. Photobiol. Sci. 10, 1056-1065 (2011).

43. Esipova, T. V. et al. Oxyphor 2P: a high-performance probe for deep-tissue longitudinal oxygen imaging. Cell Metab. 29, 736-744 (2019).

44. Jia, M. J. et al. Cherenkov-excited luminescence scanned imaging using scanned beam differencing and iterative deconvolution in dynamic plan radiation delivery in a human breast phantom geometry. Med. Phys. 46, 3067-3077 (2019).

\section{Acknowledgements}

This work was funded by the grants R01 EB024498 (to B.W.P.), R01 EB018464 and R21 EB027397 (to S.A.V.) from the National Institutes of Health as well as by shared irradiation resources from the Norris Cotton Cancer Center (funded by P30 CA023108 (to B.W.P.)). 


\section{Author contributions}

X.C. designed the experiments, analyzed all imaging data, and wrote the paper. X.C., M.J., J.R.G. and C.Y. carried out the experiments and edited the manuscript. S.J. helped to design the experiments and write the paper. E.P.L., J.R.S. and P.B. provided advice on experimental design and data analysis and edited the manuscript. S.R.A. synthesized the probe Oxyphor PtG4. S.A.V. performed photophysical measurements, oxygen calibrations, provided partial support of the work, and edited the manuscript. D.J.G. and L.A.J. advised on radiotherapy design and data interpretation and edited the manuscript. J.T. edited the manuscript. B.W.P. conceived the study, analyzed the data, provided partial support of the work, and edited the manuscript.

\section{Competing interests}

B.W.P. is a founder and president of DoseOptics LLC, which develops camera systems and software for radiotherapy imaging of Cherenkov light for dosimetry-a related application. S.A.V. has partial ownership of Oxygen Enterprises Ltd, which owns the intellectual property for dendritic phosphorescent oxygen probes technology (US Pat. No. 9,556,213; US, 2017/0137449 A1). All other authors declare no competing interests.

\section{Additional information}

Supplementary information is available for this paper at https://doi.org/10.1038/s41467020-14415-9.

Correspondence and requests for materials should be addressed to S.A.V. or B.W.P.
Peer review information Nature Communications thanks Evan C. Unger and the other, anonymous, reviewer(s) for their contribution to the peer review of this work.

Reprints and permission information is available at http://www.nature.com/reprints

Publisher's note Springer Nature remains neutral with regard to jurisdictional claims in published maps and institutional affiliations.

(c) (i)

Open Access This article is licensed under a Creative Common Attribution 4.0 International License, which permits use, sharing, adaptation, distribution and reproduction in any medium or format, as long as you give appropriate credit to the original author(s) and the source, provide a link to the Creative Commons license, and indicate if changes were made. The images or other third party material in this article are included in the article's Creative Commons license, unless indicated otherwise in a credit line to the material. If material is not included in the article's Creative Commons license and your intended use is not permitted by statutory regulation or exceeds the permitted use, you will need to obtain permission directly from the copyright holder. To view a copy of this license, visit http://creativecommons.org/ licenses/by/4.0/.

(C) The Author(s) 2020 